\title{
Association between urine albumin creatinine ratio and high sensitivity C-reactive protein in patients with type 2 diabetes mellitus
}

\author{
Sujesh Kumar N ${ }^{1, *}$, Sajitha Krishnan² \\ ${ }^{\mathbf{1}}$ Assistant Professor, Dept. of Biochemistry, SUT Academy of Medical Sciences, Trivandrum, Kerala, ${ }^{2}$ Professor, Dept. of \\ Biochemistry, Amrita Institute of Medical Sciences and Research Centre, Kochi, Kerala, India
}

*Corresponding Author:

Email: sujeshk@rocketmail.com

Received: $11^{\text {th }}$ April, 2018

Accepted: $30^{\text {th }}$ April, 2018

\begin{abstract}
Introduction: Diabetes mellitus is one of the leading causes of morbidity and mortality for its association with complications like nephropathy, neuropathy, retinopathy and cardiovascular diseases. Chronic inflammation plays an important role in the development and progression of late complications of diabetes, and diabetic nephropathy (DN) is one of the major long-term complications of diabetes mellitus.

Aims and Objectives: To determine serum high sensitivity C-reactive protein (hs-CRP) and urine albumin creatinine ratio (UACR) in patients with type $2 \mathrm{DM}$ and to evaluate the relationship of hs-CRP with UACR as markers of early kidney damage.

Materials and Methods: Cross sectional study which included 95 clinically diagnosed type 2 DM patients of age group 40 to 70 years. Spot urine microalbumin and creatinine were estimated by immunoturbidimetry and Jaffe method respectively, and the results were reported as albumin creatinine ratio $(\mathrm{mg} / \mathrm{g})$. The patients were divided into two groups, diabetic with microalbuminuria as cases and without microalbuminuria as controls. hs-CRP was estimated by immunonephelometry method. Statistical analysis was done using SPSS 20.0. Data were analysed using student ' $t$ ' test for their level of significance. Correlation between variables was carried out using the Pearson correlation co-efficient.

Results: The mean hs-CRP was higher in diabetic patients with microalbuminuria $(7.28 \pm 3.46)$ comparing to diabetic patients without microalbuminuria $(1.04 \pm 0.85)$ and was statistically significant $(\mathrm{p}<0.001)$. The Pearson correlation test showed a positive correlation between hs-CRP level and UACR level $(\mathrm{r}=0.41 ; \mathrm{P}=0.02)$.

Conclusion: Microalbuminuria was accompanied by elevated hs-CRP level suggesting activation of inflammatory pathways in development of kidney disease. So as a screening method, measurement of serum hs-CRP along with urine albumin creatinine ratio helps in diagnosing early stages of diabetic nephropathy.
\end{abstract}

Keywords: Type 2 DM, High sensitivity C-reactive protein, Urine albumin creatinine ratio, Diabetic nephropathy.

\section{Introduction}

Diabetes mellitus is one of the leading causes of morbidity and mortality for its association with complications like nephropathy, neuropathy, retinopathy and cardiovascular diseases. ${ }^{1}$ Diabetic nephropathy (DN) is one of the major long-term complications of diabetes mellitus and is the leading cause of end-stage renal disease. ${ }^{2}$ Many studies have proved that type 2 diabetes mellitus (type $2 \mathrm{DM}$ ) was frequently associated with inflammatory state. ${ }^{3,4}$ and chronic inflammation plays an important role in the development and progression of late complications of diabetes. ${ }^{5}$ Quite a few studies put forward that proinflammatory cytokines/chemokines, such as interleukin (IL)-1, IL-6, IL-8, and tumour necrosis factor-alpha (TNF-alpha), monocyte chemoattractant protein-1 (MCP-1), intercellular adhesion molecules (ICAM-1), vascular cellular adhesion molecules (VCAM-1) and C-reactive protein (CRP) play significant roles in the pathogenesis of diabetic nephropathy. ${ }^{6,7}$ Inadequate control of blood glucose level increases the risk of complications of diabetes mellitus by causing low-grade vascular inflammation and endothelial dysfunction. ${ }^{8}$
The earliest clinical evidence of renal dysfunction in patients with diabetes is generally microalbuminaria - a sign of vascular endothelial dysfunction ${ }^{9}$ and by early detection of microalbuminuria in diabetics can significantly reduce the progression of renal complications. ${ }^{10}$ High sensitivity C-reactive protein (hsCRP) is one of the sensitive markers of subclinical inflammation, and its level in serum concentration is a gauge of intensity of low-grade inflammation of the arterial wall. ${ }^{8}$ The main advantage of hs-CRP is that it can be measured at any time of the day and is also a very stable parameter. ${ }^{11}$ An understanding of the clinical pathology associated with the development and advancement of diabetic nephropathy is worthwhile for instituting effective therapeutic approaches to prevent the progression. One of the previous study stated that low-grade inflammation, reflected by high CRP levels were associated with a subsequent augmentation of urinary albumin excretion in patients with type 2 diabetes. ${ }^{12}$ The aim of the study is to determine the serum high sensitivity C-reactive protein (hs-CRP) and urine albumin creatinine ratio (UACR) in patients with type 2 diabetes mellitus and to scrutinize the role of low-grade inflammation in the pathogenesis of early kidney damage in type 2 diabetes mellitus by evaluating 
the association between hs-CRP with urine albumin creatinine ratio.

\section{Materials and Methods}

The design was a cross-sectional study conducted in a tertiary care centre for a period of seven months from January 2015 to August 2015, and was carried on patients with type 2 diabetes mellitus presented to the endocrinology clinics. The study proposal was approved by the Institutional ethical committee and informed consent was obtained from all study subjects. In the present study ninety five (95) clinically diagnosed type $2 \mathrm{DM}$ patients with more than ten years of duration in the age group of 40 to 70 years were selected. We excluded subjects with history of any significant infectious diseases, trauma, malignancy, active immunological diseases, cardiovascular disease; tuberculosis, pregnancy or confounding factors for proteinuria such as severe uncontrolled hypertension or renal insufficiency from the study. Detailed history was taken and physical examination was done for all patients who were enrolled for the study.

Spot untimed urine samples were collected for quantitation of albumin and creatinine. Urine microalbumin and creatinine were estimated by immunoturbidimetry and Jaffe method respectively, using OLYMPUS AU2700 analyser and the results were reported as urine albumin creatinine ratio $(\mathrm{mg} / \mathrm{g})$. The concentration ratio of urine albumin to creatinine was used to assess the total daily albumin excretion. Microalbuminuria was defined as urine albumin value between $30 \mathrm{mg} / \mathrm{day}$ and $300 \mathrm{mg} / \mathrm{day}$. Presence of microalbuminuria was equated as a urinary albumin: creatinine ratio of more $25 \mathrm{mg} / \mathrm{g}$ for women and more than $17 \mathrm{mg} / \mathrm{g}$ for men, which is based on the National Kidney Foundation's Kidney Disease Outcome Quality Initiative working group definition. ${ }^{13}$ High sensitivity C-reactive protein was estimated by immunonephelometry method using BECKMAN COULTER IMMAGE® Immunochemistry Systems. Analytical sensitivity for CRP determination by this assay system was $0.06 \mathrm{mg} / \mathrm{L}$. The desirable level of hsCRP is $<1 \mathrm{mg} / \mathrm{L}$. The patients were divided into two groups, diabetic with microalbuminuria as cases and without microalbuminuria as controls.

\section{Statistical Analysis}

Statistical analyses were performed using the SPSS software (Statistical Package for the Social Sciences, version 20.0, SPSS Inc, Chicago, Ill, USA). Continuous variables were expressed as mean \pm standard deviation. Data were analysed using independent student ' $\mathrm{t}$ ' test for their level of significance. Chi square test was used to determine association between micro-albuminuria and hs-CRP. Correlation between variables was carried out using the Pearson correlation co-efficient. The value of $p<0.05$ was taken as significant.

\section{Results}

The present study enrolled ninety five (95) clinically diagnosed type 2 diabetes mellitus patients. Out of ninety five (95) diabetic patients forty nine (49) patients $(51.6 \%)$ had microalbuminuria (cases), whereas forty six (46) patients $(48.4 \%)$ without microalbuminuria (controls). Fig. 1 demonstrates the percentage of diabetic patients with microalbuminuria and without microalbuminuria. Mean urine albumin creatinine ratio of diabetic patients with microalbuminuria was $134.6 \pm 56.78 \mathrm{mg} / \mathrm{g}$ and diabetic patients without microalbuminuria were $10.42+6.24$ $\mathrm{mg} / \mathrm{g}$. This difference was found to be statistically significant ( $\mathrm{p}$ value $<0.001$ ). Table 1 depicts mean value of urine albumin creatinine ratio of diabetic patients with and without microalbuminuria. Mean serum hs-CRP value of diabetic patients with microalbuminuria was $7.28 \pm 3.46 \mathrm{mg} / \mathrm{L}$ and diabetic patients without microalbuminuria was $1.04 \pm$ $0.85 \mathrm{mg} / \mathrm{L}$. This difference was also found to be statistically significant ( $\mathrm{p}$ value $<0.001)$. Table 2 depicts mean value of serum hs-CRP of diabetic patients with and without microalbuminuria. It was found that out of the forty nine patients with microalbuminuria, forty three $(87.8 \%)$ patients had associated increase of hs-CRP. In contrast, of 46 patients without microalbuminuria, $39(84.8 \%)$ had normal hs-CRP level $(\mathrm{P}<0.001)$. Table 3 depicts association between microalbuminuria and hs-CRP in diabetic patients. Correlation between variables was carried out using Pearson correlation co-efficient which showed a significant positive correlation between hsCRP level and urine albumin creatinine ratio level $(\mathrm{r}=$ $0.41 ; \mathrm{P}=0.02$ ) which is shown in Table 4. Fig. 2 demonstrates the correlation between hsCRP and urine albumin creatinine ratio level. 


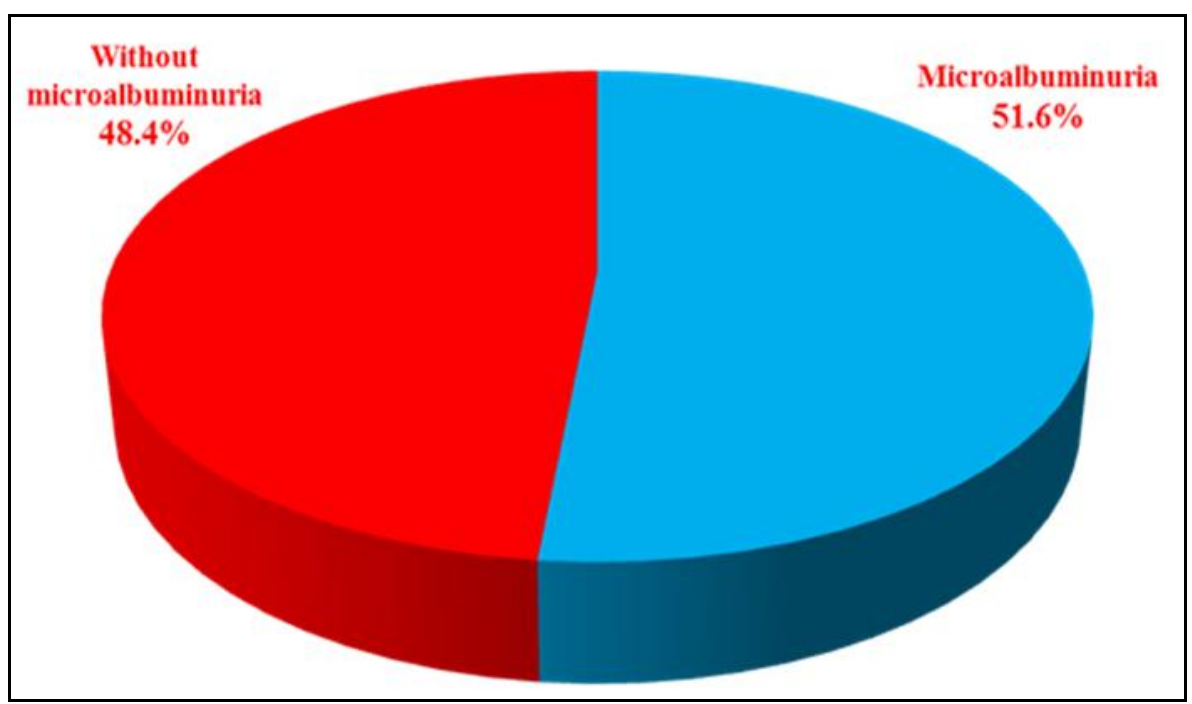

Fig. 1: Percentage of diabetic patients with microalbuminuria and without microalbuminuria

Table 1: Mean value of urine albumin creatinine ratio of diabetic patients with and without microalbuminuria

\begin{tabular}{|l|c|c|c|}
\hline & $\begin{array}{c}\text { Diabetics with } \\
\text { microalbuminuria }(\mathbf{n}=\mathbf{4 9}) \\
(\text { Mean } \pm \text { SD) }\end{array}$ & $\begin{array}{c}\text { Diabetics without } \\
\text { microalbuminuria }(\mathbf{n}=46) \\
\text { (Mean } \pm \text { SD) }\end{array}$ & p value \\
\hline $\begin{array}{l}\text { Urine albumin } \\
\text { creatinine ratio } \\
(\mathrm{mg} / \mathrm{g})\end{array}$ & $134.60 \pm 56.78$ & $10.42 \pm 6.24$ & $<0.001$ \\
\hline
\end{tabular}

Table 2: Mean value of serum hs-CRP of diabetic patients with and without microalbuminuria

\begin{tabular}{|l|c|c|c|}
\hline & $\begin{array}{c}\text { Diabetics with } \\
\text { microalbuminuria (n=49) } \\
(\text { Mean } \pm \text { SD) }\end{array}$ & $\begin{array}{c}\text { Diabetics without } \\
\text { microalbuminuria (n=46) } \\
(\text { Mean } \pm \text { SD) }\end{array}$ & p value \\
\hline hs-CRP $(\mathrm{mg} / \mathrm{L})$ & $7.28 \pm 3.46$ & $1.04 \pm 0.85$ & $<0.001$ \\
\hline
\end{tabular}

Table 3: Association between microalbuminuria and hs-CRP in diabetic patients

\begin{tabular}{|l|c|c|c|}
\hline \multicolumn{1}{|c|}{ Total no of cases $\mathbf{n = 9 5}$} & $\begin{array}{c}\text { hSCRP raised n } \\
(\mathbf{\%})\end{array}$ & hsCRP normal n (\%) & p-value \\
\hline $\begin{array}{l}\text { Diabetics with } \\
\text { microalbuminuria }(\mathrm{n}=49)\end{array}$ & $43(87.8)$ & $6(12.2)$ & \\
\hline $\begin{array}{l}\text { Diabetics without } \\
\text { microalbuminuria }(\mathrm{n}=46)\end{array}$ & $7(15.2)$ & $39(84.8)$ & $<0.001$ \\
\hline
\end{tabular}

Table 4: Correlation between urine albumin creatinine ratio and hs-CRP in diabetic patients

\begin{tabular}{|l|c|c|}
\hline \multicolumn{1}{|c|}{ Parameter } & \multicolumn{2}{|c|}{ hsCRP } \\
\hline $\begin{array}{l}\text { Urine albumin } \\
\text { creatinine ratio } \\
\text { (UACR) }\end{array}$ & 0.41 & $\mathrm{p}$ value \\
\cline { 2 - 3 } & & 0.02 \\
\hline
\end{tabular}




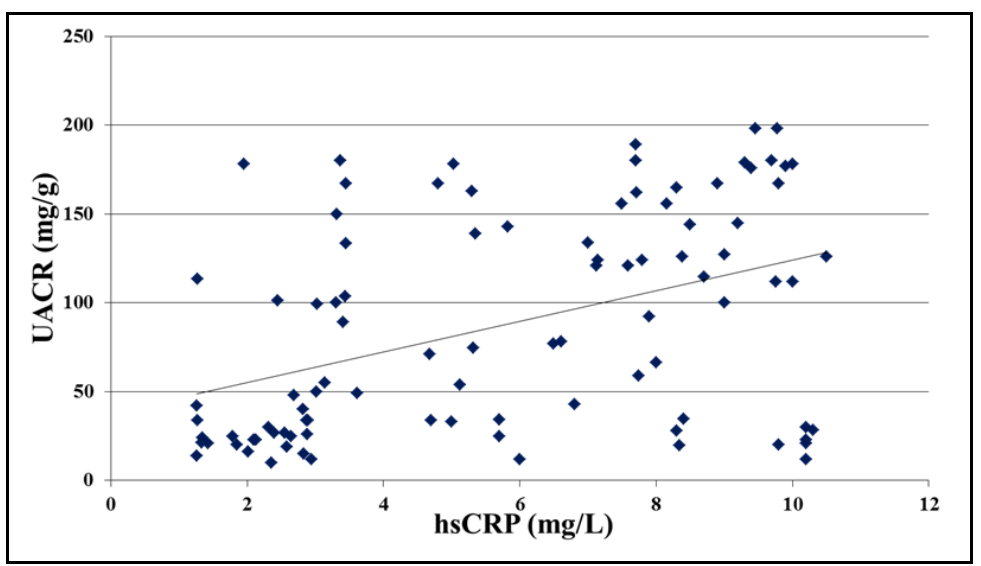

Fig. 2: Correlation between hsCRP and urine albumin creatinine ratio

\section{Discussion}

C-reactive protein (CRP) is an acute-phase reactant, and is considered as a biomarker of inflammation. Plasma CRP level increase hastily and intensely in response to tissue injury or inflammation, but its measurements in apparently healthy individuals may not truly reflect the basal level if inflammation is present. High-sensitivity CRP (hs-CRP) is more sensitive than standard CRP and is utilised for detecting normal or baseline concentrations. High-sensitivity assay techniques such as immunoturbidimetry, immunonephelometry, high-sensitivity enzyme-linked immunosorbent assay (ELISA) can sense CRP with a sensitivity range of 0.01 to $10 \mathrm{mg} / \mathrm{L}^{14}$ These highsensitivity assays help quantify low grades of systemic inflammation, in the absence of overt systemic inflammatory or immunologic disorders. The prevalence of microalbuminuria in type 2 diabetic patients in the present study is $51.6 \%$. Microalbuminuria is defined as urinary albumin excretion ranging from 30 to $300 \mathrm{mg}$ in a 24-hr urine collection. ${ }^{15}$ It is a marker of diffuse endothelial dysfunction. The suggested method to estimate albuminuria is to measure urinary albumin to creatinine ratio (UACR) in a spot urine sample. The rationale for measuring UACR is to overcome variation in urine concentration and dilution. Urinary albumin to creatinine ratio is calculated by dividing albumin concentration in milligrams by creatinine concentration in grams. Albumin excretion rate $\geq 30 \mathrm{mg} / 24$ hours or albumin to creatinine ratio (ACR) $\geq 30 \mathrm{mg} / \mathrm{g}$ is used as a marker of renal impairment. Persistent microalbuminuria is a strong predictor of development of diabetic nephropathy and lead to end stage renal disease if flouted. Therefore, detection of microalbuminuria during the early period may help to prevent progression of kidney disease in diabetic patients.

In our study we found that high sensitive CRP values were significantly higher in type 2 diabetic patients having microalbuminuria compared to diabetic patients without microalbuminuria indicating pertaining systemic inflammation in diabetic microalbuminuria patients compared to patients without microalbuminuria. Also, statistically positive correlation was seen between serum hsCRP and urinary albumin Creatinine level. In several studies, it has been reported that there is an association between serum CRP levels and microalbuminuria in diabetic patients. ${ }^{16-18}$ Many research works explained that elevated level of inflammatory cytokines and acute phase proteins can directly change glomerular function and thereby influence the development of microalbuminuria. And it is possible that increase of acute phase proteins and microalbuminuria have the same cause such as increased production of inflammatory cytokines. ${ }^{18}$ Quite a few mechanisms have been projected to explain the association between CRP and microalbuminuria. Animal studies in rat have shown that administration of CRP resulted in endothelial dysfunction and impaired vascular activity by inhibiting endothelial nitric oxide synthase. ${ }^{19}$ Diminished nitric oxide production promotes vasoconstriction, platelet activation, leucocyte adherence, impaired coagulation and vascular inflammation. ${ }^{20} \mathrm{CRP}$ also stimulates proinflammatory cytokine production ${ }^{21}$ leading to over production of matrix, mesangial cell proliferation, and augmented vascular permeability resulting in microalbuminuria. ${ }^{22}$

In our study we found that low-grade inflammation was already present in the early stage of microalbuminuria and confirmed the importance of inflammatory marker hsCRP, by way of the existence of a positive relationship between serum hsCRP with microalbuminuria in diabetic patients. These findings was in agreement with the article published by Elmarakby et $\mathrm{al}^{7}$ who reported that low-grade inflammation was already present in the early stage of diabetic nephropathy and low-grade inflammatory markers could serve in predicting initiation, and the progression of diabetic nephropathy. In 2002, Stehouwer et al. ${ }^{12}$ reported that CRP levels were associated with a subsequent increment in urinary microalbumin levels in patients with diabetes. Another 
study on patients with type 2 diabetes by Navarro et al. ${ }^{23,24}$ revealed that CRP levels were high in patients with microalbuminuria compared with those with normoalbuminuria. These observations suggest that low-grade inflammation, reflected by high serum hsCRP levels, may play a role in the pathogenesis of microalbuminuria which can be considered as a risk factor of diabetic nephropathy which was reported in many studies. ${ }^{25,26}$ The inference of attained results is revealed in the fact that simple, minimally invasive and reasonably priced tests such as the hsCRP measurement could be used for monitoring intensity of subclinical inflammation in patients with diabetes as an indicator of possible development of diabetic nephropathy. The novelty of the present study is to make an element that hsCRP estimation can be considered equivalent to UACR estimation for detecting early renal impairment in diabetic patients. Timely estimation of these sensitive biomarkers and possibly by early intervention could able to prevent further progression of renal damage in diabetic patient and reduce the number of patients with end-stage renal disease (ESRD). The scope of the present study is to make aware of this cost effective investigation (hsCRP) for primary care level treatment especially in primary health care centres, which can be utilised efficiently for early detection of diabetesinduced nephropathy.

\section{Conclusion}

It is concluded that hs-CRP is elevated in microalbuminuria patients and also there is a significant association between the level of serum hs-CRP and microalbuminuria in type 2 diabetic patients. Thus activation of inflammatory pathways in progression of kidney disease in diabetic patients as indicated by high sensitive CRP suggests that as a screening method, measurement of serum hs-CRP can be useful for diagnosis of early stages of diabetic nephropathy. We concluded that type 2 DM patients who have a significant risk factor for developing renal impairment should be regularly monitored for more sensitive biomarkers of nephropathy such as high sensitivity Creactive protein and urine albumin to creatinine ratio, to facilitate early detection of diabetes-induced nephropathy.

\section{References}

1. A.B. Patil, J. V. Ganu. High sensitive C- reactive protein and microalbumin in type 2 diabetes mellitus. Asian Pac. J. Health Sci 2014;1(4):319-321.

2. Khan MI, Usman K. Association of Hs-CRP and HbA1C with Microalbuminuria in Type-2 Diabetic patients in North India. Biomedical Research. 2012;23(3):380-384.

3. Pfützner A, Standl E, Strotmann HJ, et al. Association of high-sensitive $\mathrm{C}$-reactive protein with advanced stage beta-cell dysfunction and insulin resistance in patients with type 2 diabetes mellitus. Clin Chem Lab Med. 2006;44(5):556-60.

4. Aronson D. Hyperglycemia and the pathobiology of diabetic complications. Adv Cardiol. 2008;45:1-1.
5. Ridker PM, Cushman M, Stampfer MJ, Tracy RP, Hennekens CH. Plasma concentration of C-reactive protein and risk of developing peripheral vascular disease. Circulation. 1998;97:425-8.

6. Navarro-Gonz 'alez JF, Mora-Fern'andez C, Muros de Fuentes M, Garc' 1a-P'erez J. Inflammatory molecules and pathways in the pathogenesis of diabetic nephropathy. Nat Rev Nephrol. 2011;7:327-340.

7. Elmarakby AA, Abdelsayed R, Yao Liu J, Mozaffari MS. Inflammatory cytokines as predictive markers for early detection and progression of diabetic nephropathy. EPMAJ. 2010;1:117-129.

8. Bhowmick K, Kutty AVM, Shetty HV. Glycemic control modifies the association between microalbuminuria and C-reactive protein in type 2 diabetes mellitus. Indian $J$ Clin Biochem. 2007;22:53-9.

9. American Diabetes Association. Nephropathy in diabetes (Position Statement). Diabetes Care. 2004;27(1):79- 83.

10. Aggarwal J, Kumar M. Prevalence of microalbuminuria among rural North Indian population with diabetes mellitus and its correlation with glycosylated haemoglobin and smoking. J Clin Diagn Res. 2014; 8:CC11-3.

11. Goud BK, Nayal B, Devi OS, Devaki RN, Avinash SS, Satisha TG, Raghuveer CV. Comparison of microalbuminuria with hs-CRP and LDL levels in nondiabetic, non-hypertensive myocardial infarction patients. J Cardiovasc Dis Res. 2012;3:287-9.

12. Stehouwer CD, Gall MA, Twisk JW, Knudsen E, Emeis JJ, Parving HH. Increased urinary albumin excretion, endothelial dysfunction, and chronic low-grade inflammation in type 2 diabetes: progressive, interrelated, and independently associated with risk of death. Diabetes. 2002;51:1157-1165.

13. K/DOQI clinical practice guidelines for chronic kidney disease: evaluation, classification, and stratification. Am J Kidney Dis. 2002;39:S1-S266.

14. Roberts WL. CDC/AHA Workshop on Markers of Inflammation and Cardiovascular Disease: Application to Clinical and Public Health Practice: laboratory tests available to assess inflammation--performance and standardization: a background paper. Circulation. 2004;110:e572-6.

15. Allan Gaw, Michael J. Murphy, Rober A. Cowan. Text book of Clinical Biochemistry: Diagnosis and monitoring of diabetes mellitus. 3:61.

16. Brownlee M, Aiello LP, Friedman E, Vinik AI, Nesto $\mathrm{Rw}$, Boulton AJ. Complications of diabetes mellitus. In: Larsen PR, Kronenberg HM, Melmed S, Polonsky KS. Endocrinology.10th ed. Philadelphia: WB Saunders; 2003. p. 1509-40.

17. Mojahedi MJ, Bonakdaran S, Hami M, Sheikhian MR, Shakeri MT, Aiatollahi H. Elevated serum C-reactive protein level and microalbuminuria in patients with type 2 diabetes mellitus. Iran J Kidney Dis. 2009 Jan;3(1):12-6.

18. Festa A, D'Agostino RD, Howard G. Inflammation and microalbuminuria in nondiabetic and type 2 diabetic subjects: the insulin resistance atherosclerosis study. Kidney Int. 2000;58:1703-10.

19. Guan H, Wang P, Hui R et al. Adeno-associated virusmediated human $\mathrm{C}$-reactive protein gene delivery causes endothelial dysfunction and hypertension in rats. Clin Chem. 2009;55:274-284.

20. Verma S, Wang CH, Li SH et al. A self-fulfilling prophecy: C-reactive protein attenuates nitric oxide production and inhibits angiogenesis. Circulation. 2002;106:913-919. 
21. Verma S, Li S.H, Badiwala M.V et al. Endothelin antagonism and interleukin-6 inhibition attenuate the proatherogenic effects of C-reactive protein. Circulation. 2002;105:1890-1896.

22. Horii $\mathrm{Y}$, Iwano M, Hirata $\mathrm{E}$ et al. Role of interleukin-6 in the progression of mesangial proliferative glomerulonephritis. Kidney Inter Suppl. 1993;39:S71S75.

23. Navarro JF, Mora C, Muros M, Garca J. Urinary tumour necrosis factor-alpha excretion independently correlates with clinical markers of glomerular and tubulointerstitial injury in type 2 diabetic patients. Nephrol Dial Transplant. 2006;21:3428-3434.

24. Navarro JF, Mora C, Maca M, Garca J. Inflammatory parameters are independently associated with urinary albumin in type 2 diabetes mellitus. Am J Kidney Dis. 2003;42:53-61.

25. Mangili R. Microalbuminuria in diabetes. Clin Chem Lab Med. 1998;36:941-6.
26. Hayashino Y, Mashitani T, Tsujii S, Ishii H. Serum highsensitivity c-reactive protein levels are associated with high risk of development, not progression, of diabetic nephropathy among Japanese type 2 diabetic patients: A prospective cohort study. Diabetes Care. 2014 Nov;37(11):2947-52.

How to cite this article: Kumar SN, Krishnan S. Association between urine albumin creatinine ratio and high sensitivity $\mathrm{C}$-reactive protein in patients with type 2 diabetes mellitus. Int J Clin Biochem Res. 2018;5(3):386-391. 